\title{
EL PAPEL DEL MAESTRO EN EL DESARROLLO INTELECTUAL DE SUS ESCOLARES
}

\author{
The role of the teacher in the intellectual development of his schools
}

\author{
Diaki Mateus Alfonso, Lcdo. \\ Universida de Kimpa Vita, Angola-Uige \\ https://orcid.org/0000-0003-3204-297X \\ mateusafonsodiaki@gmail.com
}

\begin{abstract}
Palabras claves: Maestro, Inteligencia, Enriquecimiento, Desarrollo Personalidad.
\end{abstract}
Keywords: Teacher, Intelligence, Enrichment, Personality Development.
Recibido: 12 de septiembre de 2018

Aceptado: 21 de diciembre de 2018

\section{RESUMEN}

Esta contribución tiene como objetivo caracterizar el papel del maestro en el desarrollo intelectual de los escolares teniendo en cuenta su doble papel, de educador y orientador. Entre los principales referentes teóricos tomados están los de Castellano, (1997), Gardner, (1993), López, (2015), Aciego, (2017), Vygotsky, (1987) que en sus investigaciones han transcurrido más para allá en el análisis de la inteligencia humana. La metodología utilizada abarcó los métodos teóricos como el análisis-síntesis, inductivo-deductivo, revisión de artículos y ponencia sobre la temática. Tensionamos concluir viendo desde una mirada analítica la influencia que ejerce el maestro desde su ejemplo personal, motivado con su tarea de educar y a su vez preparar hombre para el futuro. Su rol de trabajar en función del enriquecimiento intelectual de los escolares para el desarrollo de su personalidad.

\section{ABSTRACT}

The purpose of this contribution is to characterize the role of the teacher in the intellectual development of schoolchildren, taking into account their dual role as educator and counselor. Among the main theoretical references taken are those of Castellano, (1997), Gardner, (1993), López, (2015), Aciego, (2017), Vygotsky, (1987) that in their research have gone further in the analysis of human intelligence. The methodology used included the theoretical methods such as analysis-synthesis, inductive-deductive, review of articles and presentation on the subject.We stress concluding seeing from an analytical perspective the influence exerted by the teacher from his personal example, motivated with his task of educating and in turn preparing man for the future. Its role to work in function of the intellectual enrichment of the students for the development of their personality. 


\section{INTRODUCCIÓN}

La inteligencia humana y su repercusión en el desarrollo intelectual de los alumnos, en los últimos tiempos, viene mereciendo mucha atención en los debates académicos y científicos; por cuanto, el desarrollo científico y tecnológico acelerado, que nos ha hecho evolucionar hasta la era de las tecnologías de información y comunicación, requiere ineludiblemente del desarrollo de la inteligencia humana y el desarrollo del intelecto.

El hombre por naturaleza es inteligente, pero en su convivio social se estimula cada vez más su inteligencia a través de actividad y la comunicación, fruto de su capacidad de adaptación e interacción con el medio social. En esto desempeña un papel importante lo que hacen los maestros en la escuela para cumplir tal propósito.

Al decir de Castellano (1997), uno de los problemas centrales de la sociedad, por el ende de la educación, es el desarrollo de la inteligencia humana y de los múltiples talentos que cristalizan las potencialidades humanas. Entre las principales fuentes de las transformaciones y del avance social se encuentra sin duda en el nivel alcanzado por desarrollo de la personalidad y capacidad humana. Escribía, en 1884, José Martí.

En el mundo, se ha tenido la experiencia de disfrutar y enriquecer estos conocimientos relativos al desarrollo intelectual, con la aportación realizada de grandes científicos e investigadores tales como, Albert Einstein (1879), Jean Piaget (1896), Charles Babbage 1791), Blaise Pascal (1623), Sigismund Freud (1856), Aristóteles (384 a.C), L Vygotsky (1896), Sócrates (399 a.C), Stephen Hawking, (1942) entre otros.

Ellos han desarrollado teorías que cambiaron el mundo para mejor, sus importantes contribuciones dieron al mundo una mirada a este contenido para potenciarlo desde diferentes perspectivas en el ámbito escolar, lo cual sigue teniendo vigencia en los días actuales. Por tanto, no se puede negar ni dejar de atender la atención y el tratamiento al enriqueciendo intelectual de los escolares con nuevos métodos, técnicas y vías, como una misión imprescindible en este siglo XXI.

La metodología utilizada para el desarrollo del artículo se basó en la utilización de métodos fundamentalmente de carácter teórico. Tales como: como el análisis-síntesis, inducción-deducción, a partir de la revisión de artículos, tesis, ponencias e investigaciones sobre esta problemática teórico metodológica, para hacer una fundamentación referencial del objeto que se aborda.

Por lo que, esta ponencia tiene como objetivo: ofrecer importantes reflexiones sobre el papel del maestro en el desarrollo intelectual de los escolares.

\section{DESARROLLO}

La teoría del desarrollo intelectual que propone Piaget, parte de la base que todos los individuos pasan irremediablemente por diferentes etapas ordenadas y progresivas, aunque eventualmente con ritmos distintos; esto no significa que todos los hacen de la misma forma, ni que todas las personas alcanzan la etapa superior. Para este investigador el desarrollo intelectual es un proceso que sigue un camino ordenado, sistemático y secuencial.

Las más viejas concepciones acerca de la naturaleza fija, predeterminada, biológica y unilateral, de las capacidades humanas, han ido cediendo su lugar a una visión mucho más profunda. Se reconoce cada vez más, que el desarrollo intelectual y el desarrollo de la inteligencia humana se estimula desarrolla en estrecha interacción con el medio, con la cultura y con la sociedad, en el sentido más amplio.

La educación, proceso permanente que capacita al hombre para la vida, cumple una función esencial en el crecimiento intelectual y personal de los individuos. El proceso de formación y despliegue de lo humano posee una naturaleza social interactiva y modificable. Vygotsky, (1987).

Al decir de Castellano, (1997), las definiciones más comunes han puesto el énfasis en la inteligencia como capacidad del hombre para pensar y desarrollar un pensamiento abstracto, como posibilidad de comprender el mundo a partir del procesamiento de la formación del entorno como capacidad de aprendizaje y de adatarse a las situaciones nuevas que el sujeto enfrenta constantemente en su vida.

Piaget, parte del punto de vista de que como todos los organismos se adaptan a su ambiente, tienen que poseer alguna forma de estructura u organización que haga posible la adaptación. Así, pues, considera que la organización y la adaptación son las invariantes fundamentales del funcionamiento.

Por otra parte, considera que el funcionamiento intelectual es tan sólo un caso especial del funcionamiento biológico especial, considera que la organización y la adaptación son esenciales también para el primero. 
Sin embargo, se reflexiona y connota que los procesos de enriquecimiento y desarrollo intelectual y socio afectivo estudian el comportamiento humano en el contexto de enseñanza-aprendizaje, tanto de tipo formal como informal, tanto en la escuela como en el estado de constante aprendizaje del ser humano Aciego, (2017).

Basado en esta perspectiva, se puede afirmar que el enriquecimiento intelectual tiene relación con la inteligencia emocional porque se asocia más a las capacidades cognitivas del ser humano, el razonamiento, la memoria, es decir, tiene que ver más con el conocimiento del mundo interior y exterior, así como, de su gestión para profundizar en el conocimiento, mientras que la inteligencia emocional, por sus características intrapersonal e interpersonal, se relaciona con la capacidad de saber cuáles son los móviles emocionales dinamizadores del conocimiento y saber gestionarlos correctamente.

Se supone que un buen educador, particularmente el maestro, debe convertirse en un líder para sus escolares portador de una eficaz inteligencia emocional, teniendo en cuenta no solo el aspecto cognitivo, sino conociendo los elementos emocionales que se proyectan a favor del desarrollo intelectual de su alumnado.

A partir de esto, el maestro en su doble papel de educador y orientador debe estimular el desarrollo de la inteligencia de sus educandos a través de variadas y creativas actividades, y con el establecimiento de una comunicación asertiva y desarrolladora que promueva y desarrolle de forma particular las capacidades, los intereses y un importante conjunto de cualidades o características personales que resultan decisivas en la conformación de su personalidad.

Es decisivo este análisis por el doble rol que desempeña el maestro como educador y como orientador de sus escolares. Lo que implica la necesidad de dominar las preferencias, intereses y potencialidades de sus educandos, para poder ejercer una influencia positiva en el desarrollo del intelecto y la inteligencia en cada uno de ellos. Además, es fundamental que el maestro haga un diagnóstico fino de sus escolares que le permita conocer las individualidades y a posterior diseñar acciones para el desarrollo de estas cualidades intelectuales.

Al hacer esto, el maestro estará determinando también el futuro profesional de los escolares, se necesita de la estimulación sistemática, oportuna y de modo creativo de las inteligencias múltiples, lo cual puede acentuarse en la etapa de la selección y elección profesional. Es importante el papel de la escuela como institución responsable en organizar las influencias internas y externas para brindar a cada escolar las herramientas necesarias para poder alcanzar el nivel del desarrollo intelectual deseado de su personalidad.

En este nuevo papel del profesorado no debe solo transmitir conocimientos sino conducir y estimular las aptitudes y potencialidades de cada educando como conductor de un grupo; un profesional que sabe sacar lo mejor de cada alumno, que lo asiste de manera optimista a resolver los conflictos en el aula, que no permite que haya alumnos aislados, indiferentes y desmotivados, que utiliza opciones, alternativas y metodologías acorde al diagnóstico que tiene de cada uno, que se caracteriza por su trato amable, respetuoso, ético y digno, capaz de implicar, comprometer y activar con más protagonismo la totalidad de los integrantes del grupo que atiende. La instrucción es fácil, estimular y desarrollar intelectualmente a los demás y motivarlos es esa dirección de manera intrínseca sólo lo pueden hacer este tipo de maestros.

Los profesionales de la educación solo pueden encontrar las causas de los problemas del desarrollo intelectual de los alumnos a partir de un buen diagnóstico y una adecuada caracterización de estos, y con una sólida formación en cómo saber emprender el desarrollo intelectual y la inteligencia, lo que es más importante, deberían poseer habilidades sociales y competencias emocionales, para saber entusiasmar, involucrar, motivar y favorecer una buena interrelación entre todos los alumnos y las alumnas.

El trabajo del maestro está revestido de gran importancia y complejidad, por eso se exige experiencia, responsabilidad, idoneidad, ejemplo personal, motivación profesional y sentido de pertenencia en la función que desempeña, su comportamiento ético, sus conocimientos, la teoría y la metodología que emplea en la labor que ejerce es vital en lo que puede enseñar a hacer a sus escolares de manera voluntaria y constructiva.

A continuación, López, (2015) sugiere dos actividades importantes para el enriquecimiento intelectual:

- Trabajar las aptitudes y las habilidades constructivas de la inteligencia.

- Trabajar una metodología que potencie el autoaprendizaje por parte del alumno.

Este autor considera que en estas dos actividades se trabajan los siguientes procesos o aptitudes: percepción, memoria, atención, estructuración espacial, pensamiento y matemática. 
No obstante, Gardner, (1993) en su teoría sobre la inteligencia, considera que existe la inteligencia múltiple en los seres humanos. El presenta una idea muy importante al afirmar que las capacidades de nuestra mente no forman parte de una sola habilidad llamada inteligencia, sino de muchas que trabajan en paralelo y que, muchas veces, son ignoradas o eclipsadas simplemente porque no las valoramos.

El autor referido, rechazaba la idea de que existiese una inteligencia unitaria capaz de ser medida por pruebas de lápiz y papel. Si los seres humanos se caracterizan por ser increíblemente versátiles a la hora de adaptarse a los nuevos retos e improvisar, ¿por qué se es tan rígido para evaluar y medir el potencial de la mente de cada ser humano? Asimismo, se cuestiona ¿por qué no reconocer que todo aquello que nos hace únicos y especiales se encuentra en habilidades mentales que no tienen que ver solo con resolver puzzles y operaciones matemáticas, sino que también involucran la sensibilidad artística o la gestión de las emociones?

El conocimiento intelectual es la apropiación de los objetos que no pueden sernos presentados por los sentidos (mediadores), a los cuales no pueden estimular. Estos objetos son los modos de ser de las cosas y sus relaciones, qué son, qué valen, por qué y para qué son, etcétera.

Es válido recordar que los conocimientos logrados a través de diversos procesos y etapas pueden servir para adquirir nuevos conocimientos; y a la vez, constituyen un andamiaje en la posesión o incorporación a nuestra vida de conocimientos dispuestos a ser actualizados. Por ello, suele llamársele saber potencial, para diferenciarlo de la utilización práctica y efectiva de esos conocimientos que se denominan saber actual. Además, recibe el nombre de saber la terminación del proceso psíquico en que se conoce o se aprehende algo.

Así pues, Gardner desarrolló la Teoría de las Inteligencias Múltiples, según la cual cada persona dispone de varios tipos de habilidades mentales que son independientes entre sí. De este modo, alguien que obtiene una puntuación de cociente intelectual muy alta podría ser muy mala en la mayoría de las inteligencias que son ignoradas por el test que ha rellenado, y alguien que ha obtenido una puntuación muy baja podría ser un genio incomprendido en otras habilidades.

Las inteligencias múltiples según Gardner son:

1. Inteligencia lingüística

2. Inteligencia lógico-matemática

3. Inteligencia espacial

4. Inteligencia musical

5. Inteligencia corporal

6. Inteligencia intrapersonal

7. Inteligencia interpersonal

8. Inteligencia naturalista

De los 8 importantes tipos de inteligencia señalados por Gardner cada ser humano desarrolla, aunque sea uno de ellos. Es importante que el maestro tenga presente que todo ser humano es potencialmente inteligente independiente de su raza, religión, nivel familiar, e nivel social, lo que en muchos casos hay que estimular ciertas destrezas y habilidades que existen en ellos.

En la cotidianidad hemos tenido que comprobar la existencia de estos 8 tipos de inteligencia ya que hemos visto genios en la música, danza, deporte, política, en las ciencias, lo que podemos convergir en la idea de que realmente existen inteligencias múltiples en los seres humanos.

El maestro en su desempeño profesional tiene el reto de estimular estos tipos de inteligencias en todos y cada uno de sus escolares desde el contexto áulico, siendo importante investigar, diagnosticar y reconocer las potencialidades y la atención a la diversidad de sus educandos, porque solo así, podrá atender a las diferencias individuales de cada uno de ellos a favor de su enriquecimiento intelectual.

Este reto debe constituirse en tarea grata, que a pesar de su complejidad puede ser posible, teniendo en cuenta las características de los escolares y sabiendo que todos vienen de un ambiente hogareño diferente, y que cada uno viene con sus influencias externas disímiles, pero es la presencia y el hacer intencionado, organizado, sistemático, sistémico y creativo del maestro fundamental dentro de la escuela para alcanzar este desafío.

Vale comentar que un día un maestro entró en un aula por primera vez, les pidió a sus escolares que le dijeran ¿cuál es el maestro que le ha marcado en su vida y por qué? todos contestaron inmediatamente de manera aleatoria y voluntaria el primer nombre que recordó, y en sus justificativas la mayoría decía porque era bueno y los enseñó. 
Puede afirmarse que cada ser humano que un día estuvo sentado en un pupitre tiene un maestro que le ha marcado tanto negativa como positivamente su vida, sobre todo porque lo estimuló a aprender y llegar a ser inteligente. El subconsciente de cada individuo guarda recuerdos de aquellos que realmente han ejercido una fuerte influencia significativa.

Es importante tener plena conciencia de que todo el maestro tiene de que dejar sus huellas positivas marcadas en la vida de sus escolares, que debe, por tanto, dar su máximo empeño en ser recordado sobre todo por su contribución a la estimulación de la inteligencia y desarrollo intelectual.

Son muchas el requerimiento puede tener en cuenta el maestro para estimular el desarrollo de la inteligencia en los educandos entre ellas se asumen:

- Dejar que los escolares manifiesten de manera espontánea sus sentimientos y emociones y saber escucharlos de manera paciente y positiva.

- Potenciar en ellos el conocimiento de sí mismo, la capacidad de saber quién es, qué puede hacer bien con ideas que le surgen y con la estimulación de la seguridad propia para alcanzarlo.

- Demostrar a los escolares la conciencia de que es una persona importante. Por ejemplo, elogiando cualquier cosa que haga bien, dar un abrazo, dedicarles un tiempo, escucharlos con atención y comprensión.

- Enseñar que en la vida a veces para alcanzar algo importante cuesta mucha voluntad y trabajo. Por lo que hay que aprender a esperar hasta que llegue el momento de tener lo que se quiere y, que mientras tanto, se puede ir planeando y consiguiendo otras cosas similares.

- Enseñar que cuando se tiene un problema, lo primero que hay que hacer es reflexionar y luego actuar de una forma tranquila, sin dañar a otros, ni exigir a otros lo que puede lograr por sí mismo

- Enseñar la importante que es motivar a sus compañeros en el hacer o trabajar en conjunto y animados está éxito

- Tener conciencia de la importancia de confiar y quererse a sí mismo para hacer lo que le gusta y saber con alegría que puede.

- Enseñarlos y estimularlos a conversar, a relacionarse adecuadamente con los demás sin agresividad, motes, ni burlas que implique lastimar a nadie.

- Dialogar mucho con los alumnos de manera amena, jocosa e instructiva.

- Felicitarlos, ante sus éxitos y estimularlos ante sus fracasos con la seguridad que puede alcanzar grandes cosas cuando se lo propone.

- Resaltar oportunamente las aptitudes que tiene cada uno, sus cualidades positivas por encima de las negativas y los objetivos alcanzados antes que las carencias.

- Saber captar los estados de ánimos, las preocupaciones o signos de insatisfacción que transmiten los alumnos, favoreciendo la comunicación con ellos y dando ánimo, opciones y alternativas para superar lo negativo de forma positiva en interacción educador-educando en un clima emocional y afectivo favorable.

Estos requerimientos deben ser intencionados e $\mathrm{n}$ el estímulo del desarrollo de las aptitudes y las habilidades constructivas de la inteligencia y el intelecto de manera general, con nuevas metodologías que potencien un aprendizaje significativo, vivencial y desarrollador, el autoaprendizaje por parte de cada escolar en el espacio áulico fundamentalmente en interrelación e interacción con sus coetáneos.

Debe trabajarse didácticamente por la estimulación de una percepción más detallada e intencional, de la memoria lógica-verbal y racional en el intento por recordar lo aprendido y transferirlo a nuevas situaciones, desde una atención voluntaria y lógica, también con la estructuración espacial, el desarrollo de un pensamiento reflexivo y productivo, apoyado en el análisis que se promueva desde las situaciones problémicas a resolver en cada asignatura y atendiendo a los niveles de desarrollo de cada escolar.

Igualmente, es importante considerar, en el trabajo del maestro, el desarrollo progresivo de la metacognición, que en muchos casos se produce lentamente en algunos escolares, ya que pasa por los logros en el funcionamiento del pensamiento, el enriquecimiento de su lenguaje, la capacidad de planificación de sus propias tareas cognitivas, la autorregulación y el control deliberado de las funciones psicológicas básicas para aprender a aprender independientemente.

Las actividades deben concebirse metodológicamente según los distintos grados de dificultad presente en cada escolar, para lo cual han de organizarse sesiones de trabajo por niveles de atención a la diversidad y de tratamiento a cada individualidad presente en el contexto donde se aprende. Le toca a cada maestro decidir qué sesión se ajusta más a cada escolar. 
En la conducción de las diferentes trayectorias cognitivas debe tenerse presente aquellas áreas curriculares en las que el escolar es más productivo y en las que es más lento, los diferentes contextos en que pueden ser estimuladas, la capacidad de razonamiento y de resolver problemas. En atención a ello, se puede mediar para la construcción de nuevas redes de conocimientos que faciliten la transferencia de conocimientos y el deseo de aprender mejor. Por lo general, las instituciones educativas han sido rígidas en sus métodos, estilos y estrategias de aprendizaje, más centrado la impartición de contenidos que en los ritmos de aprendizaje del alumnado. No siempre los objetivos de la enseñanza se han enrumbado a tono con las potencialidades, aptitudes, posibilidades y talentos presentes en cada educando en determinadas áreas del saber, para que pueda desplegarse con éxito y equilibro personal en su vida cotidiana.

Hay alumnos con una capacidad intelectual normal que manifiestan dificultades académicas, problemas en el aprendizaje y comportamiento por abandono pedagógico, por causas de los conflictos emocionales y afectivos con sus familiares, compañeros de clase, etc. Por infortunio, son muchas influencias y situaciones que pueden afectar el desarrollo intelectual e inteligente de los escolares sometidos a ambientes familiares insanos, presiones adversas por parte de sus compañeros de aula e incluso de familiares cercanos, lo que les afecta consciente y muchas ves inconscientemente su normal desarrollo psíquico.

No obstante, se hace énfasis en que el desarrollo intelectual supone estimular las estrategias y la utilización de instrumentos acorde a lo que cada escolar necesita para la construcción de sus aprendizajes, y para que pueda responder a la resolución de muchos problemas con los esquemas que ya posee. El desarrollo de su propia actividad intelectual y práctica siempre debe estar mediatizado por las condiciones educativas, bajo la ayuda o conducción profesional de su maestro.

Algunas consideraciones al maestro de cómo llevar a efecto esa ayuda a favor del desarrollo intelectual de sus escolares son las siguientes:

- Generar durante el proceso de enseñanza aprendizaje una actitud reflexiva ante la resolución de problemas, ofreciendo los pasos posibles para la resolución, evitando la impulsividad e indiferencia por lo que se aprende.

- Saber apreciar con precisión y exactitud las respuestas que da cada escolar a las interrogantes presentadas, apoyándose del error como fuente de aprendizaje.

- Ocuparse del mejoramiento de la autoestima de cada escolar durante el acto de enseñar y aprender.

- Planificar sesiones de consultas individuales, para la ejecución de tareas y situaciones problémicas que estimulen las aptitudes, inteligencias y otras potencialidades, con la aplicación de adaptaciones curriculares, según las características propias de cada escolar.

- Conocer y aplicar la metodología de la mediación para lograr el tratamiento personal y personalizado dentro de la diversidad, con la necesaria continuidad de lo que es capaz de lograr cada educando.

- Ofrecer seguimiento a los escolares a través de lo que los padres observaban en su casa y lo que son capaces de hacer solos y con la ayuda de los otros, fundamentalmente dentro del grupo escolar.

- Saber explorar efectos y estados emocionales manifiestos en cada escolar e influir en el desarrollo de un clima afectivo favorable y positivo durante el proceso de enseñanza aprendizaje.

- Presentar situaciones con diferentes niveles de complejidad que inciten la activación de las funciones del pensamiento como son analizar, sintetizar, relacionar, definir, comparar, llegar a conclusiones.

- Generar ambientes de aprendizaje que permitan la obtención de experiencias lúdicas significativas, que motiven nuevos retos, interacciones, creatividad y novedad en los escolares.

\section{CONCLUSIÓN}

Todo escolar es potencialmente inteligente, ya que los individuos sanos cuando nacen poseen potencialidades que pueden desarrollarse a través de la actividad y la comunicación en interrelación e interacción con los demás, particularmente con su maestro.

El desarrollo intelectual y de la inteligencia puede expresarse cuantitativamente y cualitativamente teniendo en cuenta las diferencias individuales. El proceso de trasformación del intelecto puede ocurrir de manera espontánea, pero máximamente a través de un proceso socialmente dirigido y controlado con fines y objetivos, desde las instituciones educativas.

Dentro de la escuela, como institución social, es el maestro el máximo representante y responsable para desarrollar, estimular, motivar, incentivar el desarrollo intelectual y la inteligencia de todos y cada uno de sus escolares. 
El proceso de enseñanza aprendizaje significativo y desarrollador de cada alumno parte de la presencia del maestro cuando es un ejemplo, por su ética, calidad de didáctica y responsabilidad ante la tarea de enseñar a prender.

\section{BIBLIOGRAFÍA}

1. Aciego, R. (2017). Enriquecimiento intelectual socio afectivo. Departamento de Psicología Educativa de la Universidad de La Laguna, recuperado de: http://periodismo.ull.es/tag/enriquecimiento-intelectual-ysocioafectivo/

2. Agencia EFE, (2001). Investigadores chinos descubren beneficios de educación de bebés, Pekín.

3. Bruner, J. (1988). Desarrollo cognitivo y educación. Madrid: Morata. S.A.

4. Castellano, D. (1997). Módulo: Enriquecimiento Intelectual en el Proceso Docente-Educativo. recuperado de : https://www.nous-mdc.net/castellano/m\%C3\%A9todo-feuerstein/programa-de-enriquecimiento-instrumentalpei/

5. Castellanos, D. (1997). Modelo Heurísitico para la Identificación del Talento en el Contexto Escolar: Una Alternativas para el Profesor. Tesis de Maestría. La Habana: Instituto Superior Pedagógico E. J. Varona.

6. Castellanos, D. y Grueiro, I. (1995). ¿Puede ser el maestro un facilitador? Una reflexión sobre la inteligencia y su desarrollo. Palacio de las Convenciones. Pedagogía 95.

7. Castellanos, D. y Grueiro, I. (1997). ¿Puede ser el maestro un facilitador? Una reflexión sobre la inteligencia y su desarrollo. Material Curso Pre-Reunión Pedagogia 97. La Habana: Palacio de las Convenciones.

8. Catellanos, D. y Córdova, D. (1992). Hacia una comprensión de la inteligencia. La Habana. Materiales. CESOFTE-VARONA.

9. Collazo, B. Puentes, M. (1992). La orientación en la actividad pedagógica. La Habana: Pueblo Educación.

10. Gardner, H (1993). Estructuras de la Mente Estructuras de la Mente, La Teoría de Las Inteligencias Múltiple. New York: Basic Book.

11. Glez,F.yMitjans, A. (1989). L a personalidad, su educación y desarrollo. La Habana. Edit. Pueblo y Educación.

12. López, N. (2015). Actividades para enriquecimiento intelectual. Posteado en 12. NEAE, 12.01. Percepción / Discriminación visual, 12.02. Orientación espacio - temporal, 12.03. Comprensión del lenguaje, 12.05. Atención, 12.06. Memoria, 12.08. Razonamiento lógico, II - Primaria Recuperado de: http://www3.gobiernodecanarias.org/medusa/ecoescuela/recursosdigitales/2015/01/26/actividades-deenriquecimiento-intelectual/.

13. Mitjans, A. (1995). Creatividad, personalidad y educación. La Habana: Pueblo y Educación.

14. Piaget, J. \&Inhelder, Barben. (2002). Psicología del Niño. (16ª . Ed.). Madrid- España:

15. Piaget, J. (1973). Psicología de la inteligmcia. Traducido del francés. Buenos Aires: Ed, Psiqué.

16. Piaget, J. (1977). EpistemologúJ plica. Traducido del francés. Buenos Aires: Solpin.

17. Piaget, J. (1980). Problemas de psicologíagmética. Traducido del francés. Barcelona: Ed. Ariel.

18. PNUD (2002). El rol de la prensa en el desarrollo humano, Programas de las Naciones Unidas para el Desarrollo, Lima, Perú.

19. PNUD (2002). Informe sobre desarrollo humano 2002, Programa de las Naciones Unidas para el Desarrollo, Nueva York, Estados Unidos.

20. RUIZ, R. (2006).Historia y evolucion del pensamiento cientifico.www.monografias.com/trabajos-pdf/historiapensamiento-cientifico/historia-pensamiento-cientifico.shtml, publicado en Internet en versión español e inglés. "algún día, cuando hayamos controlado los vientos, las olas, las mareas y la gravedad, dirigiremos a Dios las energías del amor; y entonces, por segunda vez en la historia de la humanidad, el hombre habrá descubierto el fuego". (Pierre Teilhard de Chardin). Recuperado de: www.slideshare.net/Euler/slideshows.

21. Simón, C. (1999), El cerebro de Einstein tenía más desarrollada la zona parietal inferior, Diario Médico (http://diariomedico.com/neurologia/n180699.html).

22. Vigotsky, L. (1987). Historia del desarrollo de las funcionespsíquicassuperiores. La Habana: Editorial Pueblo y Educación.

23. Vygotsky, L. (1983). Pensamiento ylenguaje. EnInvest spsicológicasescogidas (Vol. 2) Moscú: Ediciones Academia de CienciasPedagogicas. (tnruso).

24. Vygotsky, L. (1984). Historia del desarrollo de las funcionespsíquicassuperiores, EnInvestigacionesfJsicológicasescogidas (Vol. 3). Moscú: Ediciones Academia de CienciasPedagógicas. (Enruso). 